\title{
Keyword Driven Image Description Generation System
}

\author{
Sreela Sreekumaran Pillai Remadevi Amme Sumam Mary Idicula
}

Artificial Intelligence and Computer vision Lab, Department of Computer Science, Cochin University of Science And Technology, Kerala, 682022, India

\begin{tabular}{l} 
A R T I C L E I N F O \\
\hline Article history: \\
Received: 15 May, 2020 \\
Accepted: 07 July, 2020 \\
Online: 09 August, 2020 \\
\hline Keywords: \\
Image captioning \\
Keyword Extraction \\
BLSTM \\
\hline
\end{tabular}

\section{Introduction}

Image description generation is an active research area for increasing the performance of an image search engine. The system is also useful for accessing image collections, helping visually impaired people, enhancing the education system, robotics, etc.

An image is described by using objects, attributes, actions, scenes, and spatial relationships. In this process, all the keywords of an image are mapped to a sentence called a caption. The image description is created from image features. The main image features are low level and high-level features. Here the proposed system is focused on high-level features such as keywords. The main objective of the system is to develop an image description generation system from keywords. Keyword-based image description system is a direct generation model which extracts keywords from the visual content of the image and generate sentence from keywords.

To achieve the objective, different phases are developed such as

- Keyword extraction system: The objects, attributes, actions, and scenes are extracted.

- Caption generation system: Three methods are employed for generating captions such as template-based, CFG based, and BLSTM based.

The outline of the paper is as follows. Section 2 describes the related work of the system. Section 3 explains the proposed method of the system. Section 4 discusses the experiments and results of the system. The paper is concluded in section 5 .

\section{Related works}

From the literature, there are three kinds of image description generation models such as Direct generation models, Visual space model, and Multimodal space model.

Direct generation models analyse the visual content of the image and create a sentence reflecting the meaning of the visual content. Examples of these models are Google's Neural Image Caption Generator [1], BabyTalk [2], Midge [3], Karpathy's system [4] etc. The visual space model finds similar images of the query image from a visual space and transfer the description to the query image. Research [5]-[6] follows visual space model. The multimodal space model discovers similar images from visual and textual modal space, and it is treated as a retrieval problem. While, [7]-[9] systems follow Multimodal space model.

Another classification of image description generation system is Template based approach and Deep Neural Network based approach [10]-[13]. Deep Neural Network based Image captioning systems are improved using visual attention mechanisms. The first attention-based image captioning system is done by [14]. While [15]-[17] are also proposed attention-based image captioning system. While [17]-[19] follows attention-based architecture. Examples of keyword-based image captioning systems are Midge, BabyTalk, etc. Midge and BabyTalk have less accuracy because of incorrect object detection, invalid action classification, etc. So a novel system for keyword-based image captioning systems is developed using a new keyword extraction system.

*Corresponding Author: Sreela Sreekumaran Pillai Remadevi Amma, +919497708518 \& sreela148@ cusat.ac.in 


\section{Proposed Method}

An image is processed by Computer vision algorithms can be represented as a quadruple $\left\langle O_{i}\right.$, Attr $\left._{i}, A_{i}, S_{i}\right\rangle$. Where $O_{i}$ is the set of objects in the image, Attri is the set of attributes of each object, $A_{i}$ is the set of actions of the object, and $S_{i}$ is the set of scenes associated with an image. Similarly, the description of the image can be characterized as a quadruple $\left\langle N_{d}, A d j_{d}, V_{d}, N S c_{d}\right\rangle$. Where $N_{d}$ is the set of nouns in the description, $A d j_{d}$ is the set of adjectives in the description, $V_{d}$ is the set of verbs and $N S c_{d}$ is the set of nouns associated with a scene. Image description generation is the process of mapping image quadruple $\left\langle O_{i}, A t t r_{i}, A_{i}, S_{i}\right\rangle$ to description quadruple $\left\langle N_{d}, A d j_{d}, V_{d}, N S c_{d}\right\rangle$.

The overview of the proposed methodology is explained in figure 1 . The methodology is as follows: 1) Detect Objects and scenes in the image. 2) Recognize action in an image 3) Identify attributes such as cardinality, shape, and color of the object.4) Generate text corresponding to the image. The method is divided into two parts, such as keywords generation and caption generation.

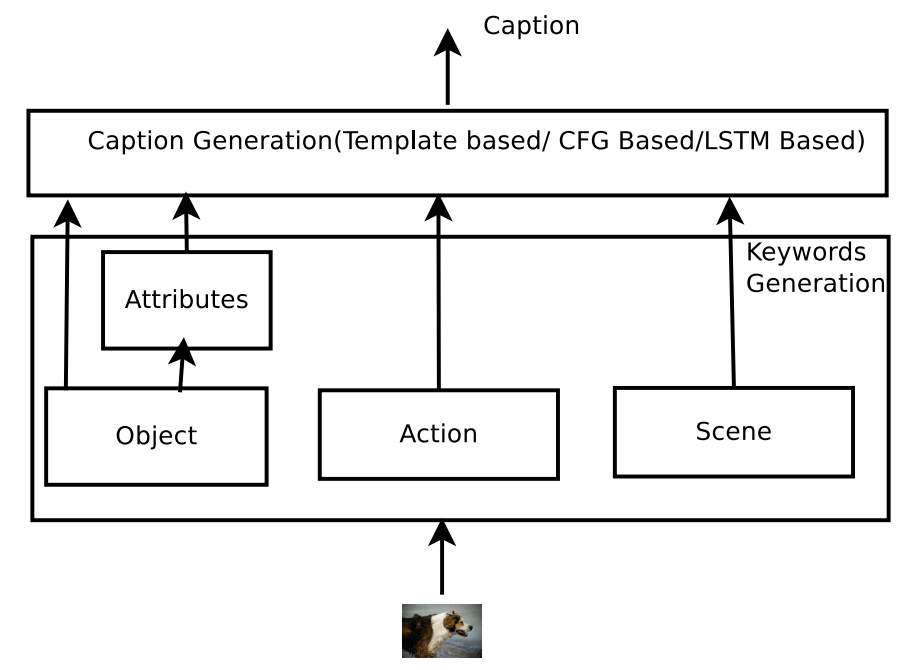

Figure 1: Proposed Architecture

\subsection{Keywords Generation}

\subsubsection{Object detection}

Darknet architecture is used for object detection. The images are divided into $7 \times 7$ grid cells. For each grid cell, we apply a classifier for identifying an object. The number of layers in Darknet is 53 . Object classes are obtained in this phase. The object is considered as the noun of the sentence. Top-k objects are selected for sentence construction.

\subsubsection{Attributes Identification}

This phase identifies the color, shape, and cardinality of the object. Attributes are identified as the adjectives of the sentence.

Color detection The color of the object is calculated from the minimum distance between $\mathrm{L}^{*} \mathrm{a} * \mathrm{~b}$ value and the average of the intensity of the image. The color with the smallest distance is treated as the color of the object.
Shape detection The algorithm for shape detection is explained below:

Compute the contour perimeter of the image;

Approximate the contour shape to another shape with fewer vertices depending upon the precision using the

Douglas-Peucker algorithm;

if The approximated shape has three vertices then

$$
\text { it is a triangle; }
$$

else

if the shape has four vertices then

Compute the bounding box of contour and find the aspect ratio;

if the aspect ratio is approximately equal to one then The shape is a square;

else

otherwise, it is a rectangle;

end

else

if the shape has more than four vertices then

end it is treated as a circle;

end

end

Cardinality Cardinality is obtained by the count of each object in the image.

\subsubsection{Action recognition}

Action recognition is done in still images. Action is treated as the verb of the sentence or caption. The architecture of action recognition is explained in figure 2 .

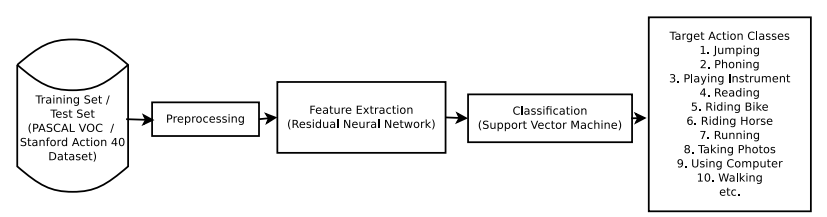

Figure 2: Proposed Architecture of action recognition

It is done using deep convolutional neural network features and Support Vector Machine. Residual Neural Network[20] is used for CNN feature extraction, and classification is done using Support Vector Machine.

\subsubsection{Scene Classification}

Context or scene is important for caption generation. It is obtained using deep neural network-based learning. The scene is the place where an image is associated. VGG architecture is used for scene classification. Three hundred sixty-five scene categories are used for this classification.

\subsection{Caption generation}

It is a process of generating sentences or captions from a set of keywords like objects, attributes, action, and scene. We implemented 
three kinds of caption generation, such as template-based, CFG based, and Bidirectional LSTM[21] based caption generation.

\subsubsection{Template based}

Keywords are extracted as a five-tuple $\langle$ object, cardinality, attribute, action, scene $\rangle$. Captions are generated from the five-tuple. Captions have a common form "There is cardinality attribute object action in the scene. The object is treated as noun, cardinality, and attributes are adjectives, the action is the verb. An example of a sentence is, "There is two blue birds fly in the sky". The set of words in the meaning representation is fixed, and generation must make use of all given content words; and, generation may insert only gluing words (i.e., function words such as there, is, the, etc.). Advantages of this approach is that it incorporates cardinality and actions in the image captions. Disadvantages of this approach is that it generates similar kinds of captions. And also still image based action recognition generates 10 classes only.

\subsubsection{CFG based}

We design a CFG for the caption. CFG has the form represented in figure 3 Using this CFG, captions are generated.

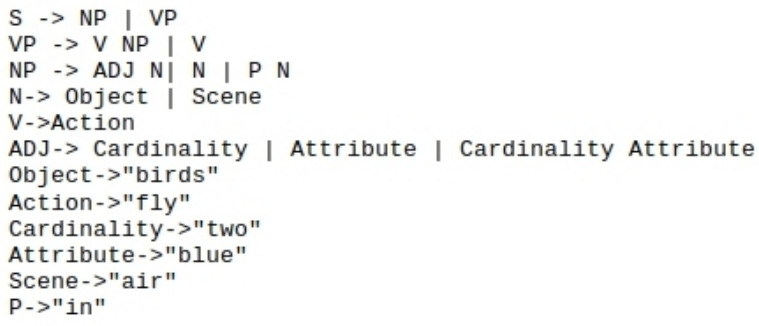

Figure 3: CFG for caption generation

\subsubsection{Bidirectional LSTM based}

It uses encoder-decoder architecture as Machine translation. Let $(k, C)$ be a keyword and caption sentence pair. Let $k=k_{1}, k_{2}, k_{3} \ldots k_{m}$ be a sequence of $\mathrm{M}$ symbols in keywords and $C=C_{1}, C_{2}, C 3 \ldots . C_{N}$ be the sequence of $\mathrm{N}$ symbols in the target caption of the image. The model maximizes the following objective.

$$
\theta^{*}=\operatorname{argmax}_{\theta} \Sigma_{k, C} \log p(C / k ; \theta)
$$

The encoder is simply a function of the following form

$$
K_{1}, K_{2}, K 3 \ldots K_{M}=\text { encoder } B L S T M\left(k_{1}, k_{2}, k_{3} \ldots k_{m}\right)
$$

Where $K_{1}, K_{2}, K_{3}, \ldots K_{M}$ is a list of hidden representation of keywords of size M. enoderBLSTM is a bidirectional LSTM. Using chain rule, the MAP of the sequence $p(C / k)$ can be decomposed as

$$
\begin{array}{r}
p(C / k)=p\left(C / k_{1}, k_{2}, k_{3}, \ldots k_{m}\right) \\
=\prod_{i=1}^{N} P\left(C_{i} / C_{0}, C_{1}, C_{2} \ldots C_{i-1} ; k_{1}, k_{2}, k_{3} \ldots k_{m}\right) \\
=\Sigma_{i=1}^{N} \log P\left(C_{i} / C_{0}, C_{1}, C_{2} \ldots C_{i-1} ; k_{1}, k_{2}, k_{3} \ldots k_{m}\right)
\end{array}
$$

where $C_{0}$ is a special "beginning of sentence" symbol. During inference, we calculate the probability of next symbol given the source encoding and the decoded target caption so far as $p\left(C_{i} / C_{0}, C_{1}, \ldots C_{i-1} ; K_{1}, K_{2}, \ldots K_{M}\right)$.

Our decoder is organized as a combination of a fully connected neural network(FCN) layer and a softmax layer. The FCN layer produces the hidden representation of the next symbol to be predicted;which then goes through the softmax layer to generate a probability distribution over candidate vocabulary symbols.

The deep neural network architecture is drawn in Figure 4.

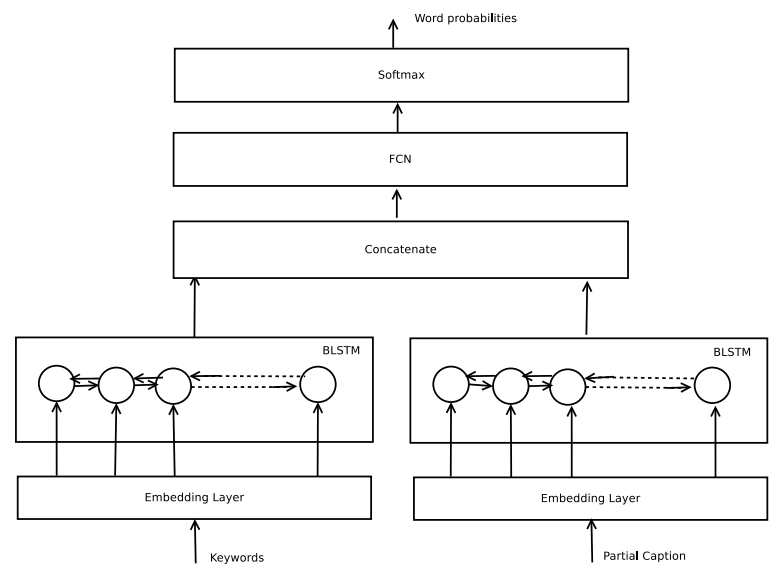

Figure 4: Bidirectional LSTM based caption generation

\section{Results}

\subsection{Keyword Extraction}

The Table 1 shows the output of keyword extraction. The table contains objects, attributes such as cardinality, shape, and color, scene, and action of images.

The Table 2 is described the various advantages and disadvantages of various phases of keyword extraction system.

\subsection{Caption Generation}

The deep neural network based caption generation model is experimented with LSTM[22] and Bidirectional LSTM. From the experiment, the accuracy graphs are plotted in figures 5 and 6 . The accuracy plot explained that the accuracy of the model is more when the bidirectional LSTM is used.

The loss of the model is plotted in figures 7 and 8 From the plots, the loss of the model is less when Bidirectional LSTM is used.

Bidirectional LSTM is more suitable for designing the caption generation from keywords. The table 3 describes the captions from keywords using three methods.

The Table 4 explains the various properties of different methods used for caption generation.

\subsection{Analysis}

The computational complexity of the caption generation from keywords is small. The space complexity of this system is described by

$$
s_{k}=\mid \text { Keywords } \mid \times \text { max_cap_len }
$$


Table 1: Keywords Extraction results

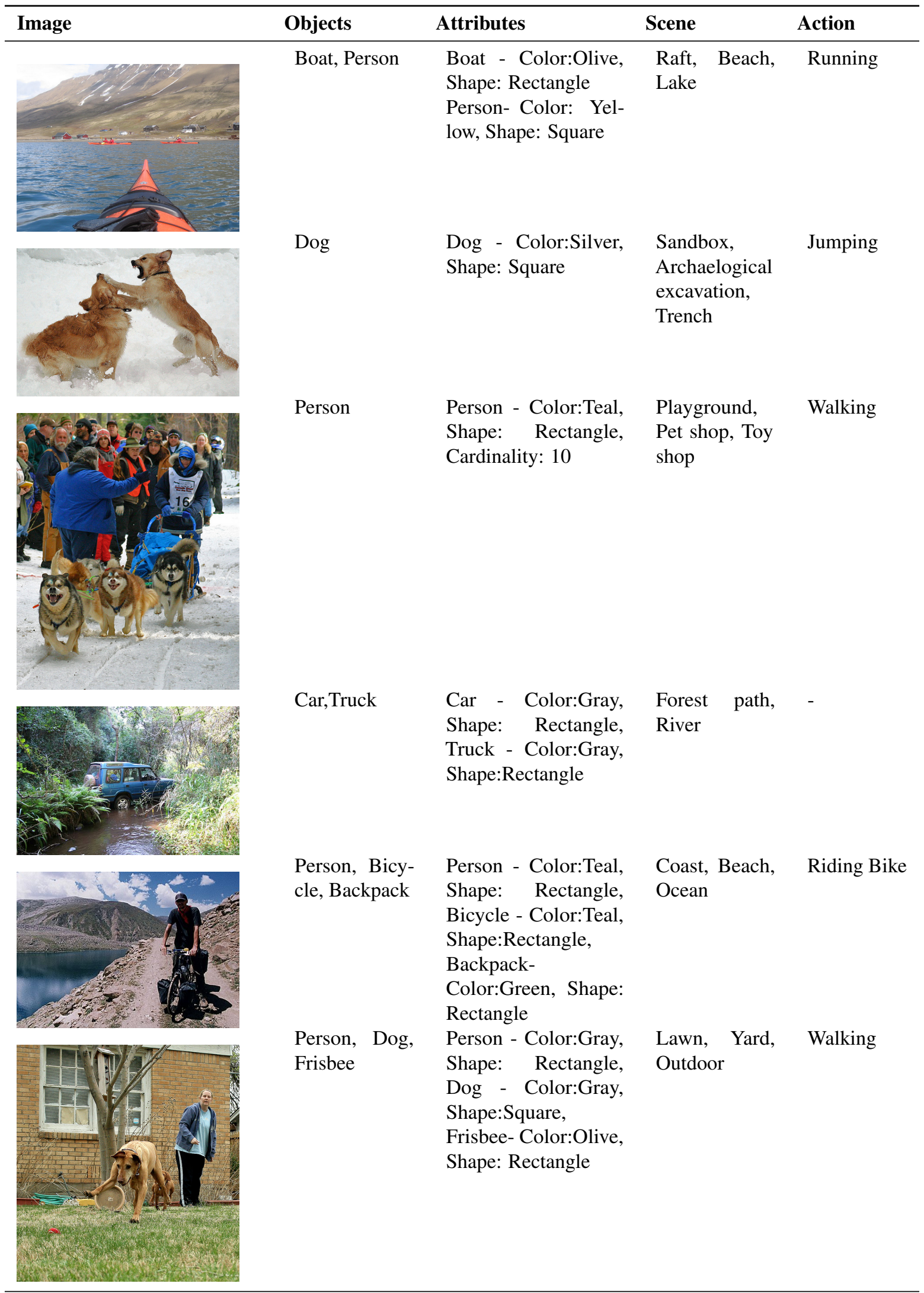


Table 2: The characteristics of different phases of Keywords extraction

\begin{tabular}{|c|c|c|}
\hline Model & Advantages & Limitations \\
\hline $\begin{array}{l}\text { Object detec- } \\
\text { tion }\end{array}$ & $\begin{array}{l}\text { Number of ob- } \\
\text { jects is } 1000 \text {. }\end{array}$ & $\begin{array}{l}\text { Vocabulary of } \\
\text { objects is lim- } \\
\text { ited }\end{array}$ \\
\hline $\begin{array}{l}\text { Attributes } \\
\text { identification }\end{array}$ & $\begin{array}{l}\text { Color, shape } \\
\text { and cardinal- } \\
\text { ity are identi- } \\
\text { fied. }\end{array}$ & $\begin{array}{l}\text { Only three at- } \\
\text { tributes of ob- } \\
\text { jects are de- } \\
\text { tected. }\end{array}$ \\
\hline $\begin{array}{l}\text { Action recog- } \\
\text { nition }\end{array}$ & $\begin{array}{l}\text { Number of ac- } \\
\text { tion classes is } \\
10\end{array}$ & $\begin{array}{l}\text { The vocabu- } \\
\text { lary of actions }\end{array}$ \\
\hline $\begin{array}{l}\text { Scene classifi- } \\
\text { cation }\end{array}$ & $\begin{array}{l}10 . \\
365 \text { scenes are } \\
\text { classified. }\end{array}$ & $\begin{array}{l}\text { is limited. } \\
\text { Number of } \\
\text { scenes is } \\
\text { limited. }\end{array}$ \\
\hline
\end{tabular}

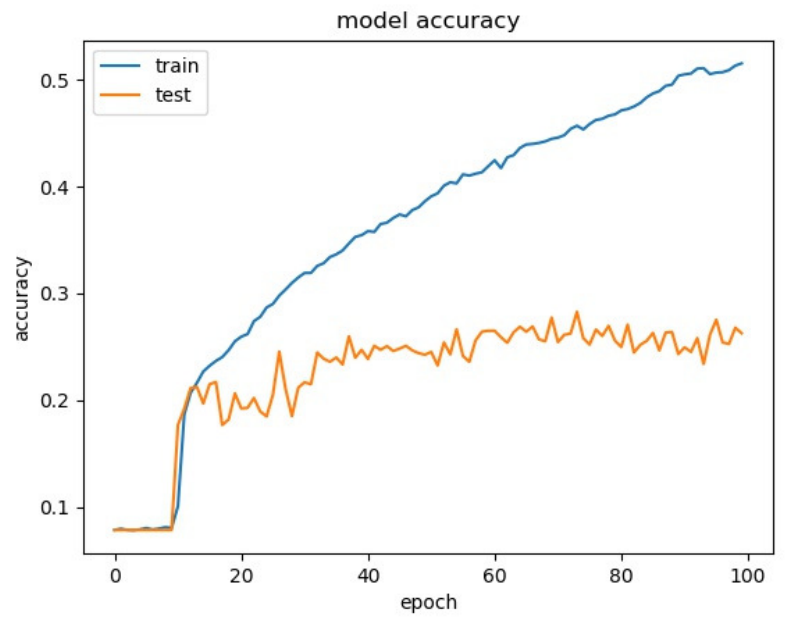

Figure 5: Accuracy plot for LSTM Based Caption generation from keywords

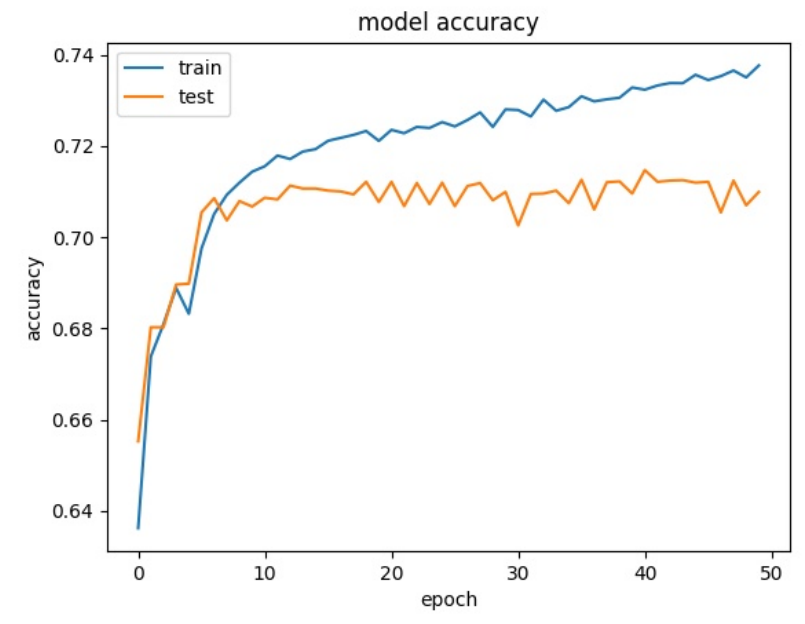

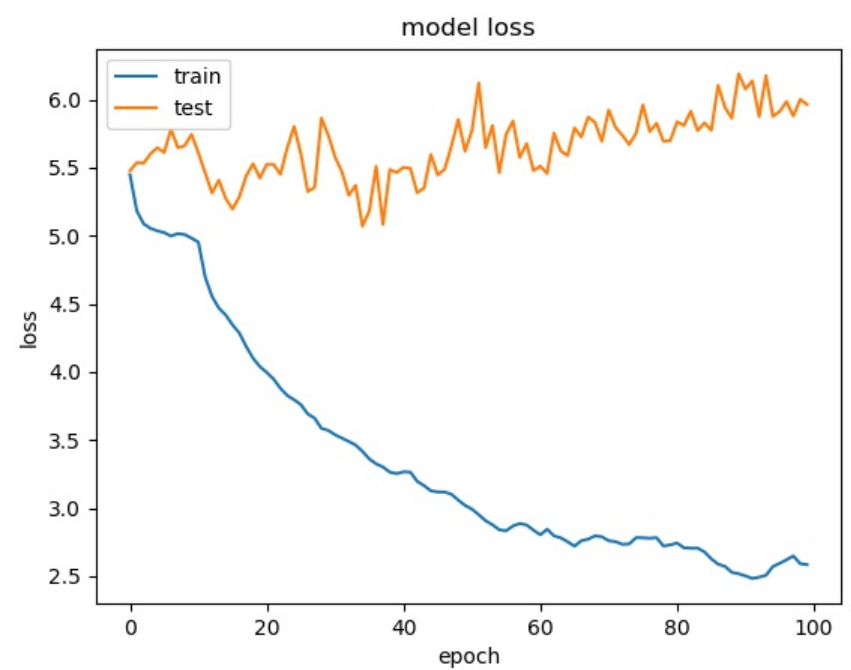

Figure 7: Loss plot for LSTM Based Caption generation from keywords

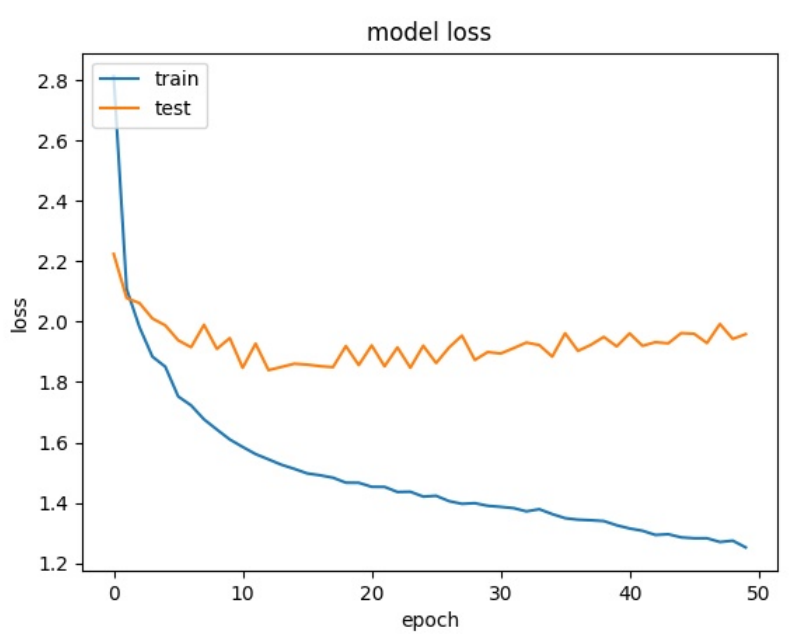

Figure 6: Accuracy plot for Bidirectional LSTM Based Caption generation from keywords
Figure 8: Loss plot for Bidirectional LSTM Based Caption generation from keywords 
Table 3: Output of caption generation from keywords using different methods.

\begin{tabular}{|c|c|c|c|}
\hline Keywords & Template based & CFG based & LSTM based \\
\hline $\begin{array}{l}\text { Person, Boat, } \\
\text { Lake, } \\
\text { ning }\end{array}$ & $\begin{array}{l}\text { There is a per- } \\
\text { son running in } \\
\text { a lake. }\end{array}$ & $\begin{array}{l}\text { A person run- } \\
\text { ning in a lake. }\end{array}$ & $\begin{array}{l}\text { A Person is } \\
\text { running in the } \\
\text { lake with a }\end{array}$ \\
\hline $\begin{array}{l}\text { Dog, Silver, } \\
\text { Sandbox, } \\
\text { Jumping }\end{array}$ & $\begin{array}{l}\text { There is a sil- } \\
\text { ver dog jump- } \\
\text { ing in sand- } \\
\text { box. }\end{array}$ & $\begin{array}{ll}\text { Silver dog } & \text { dog } \\
\text { jumping } & \text { in } \\
\text { sandbox. } & \end{array}$ & $\begin{array}{l}\text { boat. } \\
\text { Silver dog is } \\
\text { jumping in the } \\
\text { sandbox. }\end{array}$ \\
\hline $\begin{array}{l}\text { Person, Ten, } \\
\text { Playground, } \\
\text { Walking }\end{array}$ & $\begin{array}{l}\text { There is ten } \\
\text { persons walk- } \\
\text { ing in play- } \\
\text { ground. }\end{array}$ & $\begin{array}{l}\text { Ten persons } \\
\text { walking in } \\
\text { playground. }\end{array}$ & $\begin{array}{l}\text { Ten persons } \\
\text { are walk- } \\
\text { ing in the } \\
\text { playground. }\end{array}$ \\
\hline $\begin{array}{l}\text { Truck, Gray, } \\
\text { Forest path }\end{array}$ & $\begin{array}{l}\text { There is a gray } \\
\text { truck in forest } \\
\text { path. }\end{array}$ & $\begin{array}{l}\text { Gray truck in } \\
\text { forest path. }\end{array}$ & $\begin{array}{l}\text { Gray truck } \\
\text { in the forest } \\
\text { path. }\end{array}$ \\
\hline $\begin{array}{l}\text { Person, Bike, } \\
\text { Backpack, } \\
\text { Green, Coast, } \\
\text { Riding bike }\end{array}$ & $\begin{array}{l}\text { There is a per- } \\
\text { son riding a } \\
\text { bike in bike. }\end{array}$ & $\begin{array}{l}\text { Person riding } \\
\text { a bike in a } \\
\text { bike. }\end{array}$ & $\begin{array}{l}\text { Person is rid- } \\
\text { ing bike. }\end{array}$ \\
\hline
\end{tabular}

Table 4: The characteristics of different methods of caption generation

\begin{tabular}{lll}
\hline Method & Advantages & Disadvantages \\
\hline Template based & $\begin{array}{l}\text { It incorporates cardi- } \\
\text { nality and actions in } \\
\text { the image captions. }\end{array}$ & $\begin{array}{l}\text { It generates similar } \\
\text { kinds of captions. }\end{array}$ \\
CFG based & $\begin{array}{l}\text { It generates different } \\
\text { types of captions. }\end{array}$ & $\begin{array}{l}\text { It generates caption } \\
\text { with same grammar. }\end{array}$ \\
BLSTM based & $\begin{array}{l}\text { It creates various } \\
\text { kinds of captions. }\end{array}$ & $\begin{array}{l}\text { The vocabulary of ob- } \\
\text { jects, attributes, ac- } \\
\text { tions and scenes is } \\
\end{array}$ \\
& & limited. \\
\hline
\end{tabular}

where $\mid$ Keywords $\mid$ is the number of keywords and max_cap_len is the maximum caption length. The $\mid$ Keywords $\mid$ is less than the maximum number of keywords. Here we set the maximum number of keywords as 10 .

The space complexity of the caption generation in CNN featurebased caption generation system is given by

$s_{c}=\mid C N N_{-}$feature $\mid \times$max_cap_len where $\mid C N N_{-}$feature $\mid$is the length of the $\mathrm{CNN}$ feature, and it is 2208 if the CNN used is Densenet. From the analysis, $s_{k}<s_{c}$.

If the number of network parameters of the keyword-based caption generation system is $p_{k}$ and the number of network parameters of the CNN feature-based caption generation system is $p_{c}$, then $p_{k}<p_{c}$. The network of keyword-based caption generation system is small.

The limitation of the system is that the performance of the system depends on the keyword extraction system. Keyword extraction is based on object detection, object identification, action recognition, and scene classification. The mean average precision (MAP) of the action recognition system is only $66 \%$. The vocabulary of actions contains only ten words. The vocabulary size of objects is 1000 . The number of scenes is 365 . So the total vocabulary contains around $1.5 \mathrm{k}$ words. But the vocabulary size of the flickr8k dataset is 8256 . Hence the vocabulary of the keyword-based caption generation system is small.

In the future, we will concentrate on increasing the performance of the keyword extraction system. Thereby we can improve the functioning of caption generation from keywords.

\section{Conclusions}

The paper proposed a novel system for keyword-based image caption generation. The main components of the system are the keyword extraction and caption generation. It produces meaningful captions for images. The space and computational complexity of this system are small. The limitation of the system is that the performance of the system mainly depends on the keyword extraction system. So in the future, we will focus on improving the performance of the keyword extraction system. 


\section{References}

[1] Vinyals, Oriol, A. Toshev, S. Bengio, and D. Erhan. "Show and tell: A neural image caption generator." In Proceedings of the IEEE conference on computer vision and pattern recognition, pp. 3156-3164. 2015. DOI: 10.1109/CVPR.2015.7298935

[2] G. Kulkarni, V. Premraj, V. Ordonez, S. Dhar, S. Li, Y. Choi, A.C. Berg, and T.L. Berg. "Baby talk: Understanding and generating simple image descriptions.", IEEE Transactions on Pattern Analysis and Machine Intelligence, 35(12), 28912903, 2013. DOI: 10.1109/TPAMI.2012.162

[3] M. Mitchell, J. Dodge, A. Goyal, K. Yamaguchi, K. Stratos, A. Mensch, A. Berg, X. Han, T. Berg, and O. Health., "Midge: Generating Image Descriptions From Computer Vision Detections.”, Eacl, pp. 747-756, 2012.

[4] K., Andrej, and L. Fei-Fei. "Deep visual-semantic alignments for generating image descriptions." In Proceedings of the IEEE conference on computer vision and pattern recognition, pp. 3128-3137. 2015. DOI: 10.1109/CVPR.2015.7298932

[5] V. Ordonez, G. Kulkarni, and T.L. Berg., "Im2text: Describing images using 1 million captioned photographs.", Advances in Neural Information, pp. 19, 2011.

[6] P. Kuznetsova, V. Ordonez, A.C. Berg, T.L. Berg, and Y. Choi, "Collective generation of natural image descriptions.", In Proceedings of the 50th Annual Meeting of the Association for Computational Linguistics: Long Papers 1. Association for Computational Linguistics, 359-368, 2012.

[7] H., Micah, P. Young, and J. Hockenmaier, "Framing image description as a ranking task: Data, models and evaluation metrics.", Journal of Artificial Intelligence Research 47 853-899, 2013. https://doi.org/10.1613/jair.3994

[8] Gong, Yunchao, et al. "Improving image-sentence embeddings using large weakly annotated photo collections.", European Conference on Computer Vision. Springer, Cham, 2014. DOI: https://doi.org/10.1007/978-3-319-105932.35

[9] R. Socher., A. Karpathy, Q. V. Le, C. D. Manning, A. Ng, "Grounded Compositional Semantics for Finding and Describing Images with Sentences.", Transactions of the Association for Computational Linguistics (2), 207218, 2014. DOI:10.1162/tacl_a_00177

[10] Y. H. Tan and C. S. Chan, "phi-LSTM: A Phrase-Based Hierarchical LSTM Model for Image Captioning." Cham: Springer International Publishing, pp. 101-117, 2017. https://doi.org/10.1007/978-3-319-54193-8_7
[11] Tan, Y. Hua, and C.S. Chan. "Phrase-based Image Captioning with Hierarchical LSTM Model.” 2017. DOI: arXiv preprint arXiv:1711.05557

[12] Aneja, Jyoti, Aditya Deshpande, and Alexander G. Schwing. "Convolutional image captioning."Proceedings of the IEEE Conference on Computer Vision and Pattern Recognition, 2018. DOI: 10.1109/CVPR.2018.00583

[13] Han, Meng, W. Chen, and A.D. Moges. "Fast image captioning using LSTM." Cluster Computing. DOI: https://doi.org/10.1007/s10586-018-1885-9

[14] Xu, Kelvin, et al., "Show, attend and tell: Neural image caption generation with visual attention.”, International Conference on Machine Learning, 2015.

[15] Junqi Jin, Kun Fu, Runpeng Cui, Fei Sha, and Changshui Zhang. 2015. "Aligning where to see and what to tell: image caption with region-based attention and scene factorization." arXiv preprint arXiv:1506.06272, .

[16] Z.Y.Y.Y.Y. Wu and R.S.W.W. Cohen, "Encode, Review, and Decode: Reviewer Module for Caption Generation." In 30th Conference on Neural Image Processing System(NIPS), 2016.

[17] Lu, Jiasen, et al. "Knowing when to look: Adaptive attention via a visual sentinel for image captioning." Proceedings of the IEEE Conference on Computer Vision and Pattern Recognition (CVPR). 6 2017. DOI: 10.1109/CVPR.2017.345

[18] You, Quanzeng, et al. "Image captioning with semantic attention." Proceedings of the IEEE Conference on Computer Vision and Pattern Recognition, 2016. DOI: $10.1109 / C V P R .2016 .503$

[19] $\mathrm{He}$, Chen, and Haifeng Hu. "Image captioning with text-based visual attention." Neural Processing Letters 49.1: 177-185, 2019. DOI: https://doi.org/10.1007/s11063-018-9807-7

[20] He, Kaiming; Zhang, Xiangyu; Ren, Shaoqing; Sun, Jian, "Deep Residual Learning for Image Recognition". Proc. Computer Vision and Pattern Recognition (CVPR), IEEE, 2016. DOI: 10.1109/CVPR.2016.90

[21] Wang, Cheng, et al, "Image captioning with deep bidirectional LSTMs", Proceedings of the 2016 ACM on Multimedia Conference, ACM, 2016. DOI: https://doi.org/10.1145/2964284.2964299

[22] Hochreiter, Sepp, and Jrgen Schmidhuber. "Long short-term memory.", Neural computation pp. 1735-1780, $1997 . \quad$ DOI: https://doi.org/10.1162/neco.1997.9.8.1735 